\title{
Prism Signal Processing for Sensor Condition Monitoring
}

\author{
Manus Henry \\ Department of Engineering Science \\ University of Oxford \\ Oxford, OX1 3PJ, UK. \\ manus.henry@eng.ox.ac.uk
}

\author{
Oleg Yu. Bushuev, Olga L. Ibryaeva, \\ South Ural State University (SUSU) \\ Chelyabinsk, Russia. \\ oleg.bushuev@susu.ru
}

\begin{abstract}
This paper introduces a new technique, Prism signal processing, which may be used for the tracking of one or more noisy sinusoids in a signal. A simulation study is presented demonstrating the potential of Prism signal processing as an alternative to Prony's method for analyzing exponentially decaying sinusoids. One application is to sensor condition monitoring of an industrial pressure sensor, using ultrasonic excitation to evaluate the sensor's structural integrity. Initial experimental results suggest the Prism technique can reveal details in the resulting frequency/amplitude time series of each component, which is not available through Prony's method.
\end{abstract}

Keywords - pressure transducer, fault detection and diagnosis, self-validation, Prony's method, Prism signal processing

\section{INTRODUCTION}

The Internet of Things (IoT) [1] and Industrie 4.0 [2] propose substantial increases in the deployment of sensors for collecting, processing and communicating measurement data in real time - in diverse working environments. According to IBM's vision of Autonomic Computing [3], key attributes of systems with advanced capabilities include self-configuration, self-optimisation, self-healing and self-protection. In the context of sensors, many of these attributes are inherently encapsulated in the concept of the self-validating [4] or metrological self-checking [5] sensor. Here, the sensor has inbuilt capabilities - for example additional transducers and/or signal processing functions - to enable not merely the detection of the most common faults modes, but also the correction of each of the measurement values for the influence of the fault mode. In addition, the quality of the resulting measurement, whether or not a fault has occurred, is reported using standard, device-independent metrics, such as on-line measurement (metrological) uncertainty. While these requirements are ambitious, both the communication and computational bandwidth constraints of the IoT require that "the signal will be processed entirely [at] the Point of Acquisition (PoA) [6]" (i.e. within the sensor itself), and so the requirement for selfvalidation is arguably an inherent necessity.

Accordingly, there is continuing research interest both in the generation of additional diagnostic data, as well as in the

The work was supported by Act 211 Government of the Russian Federation, contract № 02.A03.21.0011 corresponding signal processing techniques needed to provide quantified diagnostic information. Naturally enough, diagnostic mechanisms are often closely aligned to the underlying measurement technology, and a number of sensor validation schemes have been developed for different sensing techniques [7], including pressure sensors [8].

The authors at SUSU have previously developed a selfvalidating pressure sensor in which an ultrasonic pulse and detection mechanism, built into the sensor body, is used to validate the mechanical integrity of the device [9]. The resulting received signal consists of a combination of exponentially decaying sinusoids, where the frequencies, initial amplitudes and rates of decay can provide diagnostic information. Extracting the parameter values of each sinusoid from the single, noisy, ultrasonic signal is challenging, and the SUSU authors have reported a modification to Prony's method in which the sample rate is selected to optimize the accuracy of the frequency measurement for each signal component [10]. Meanwhile, the Oxford author has developed a new signal processing technique suited for tracking one or more sinusoidal components in real-time data, called the Prism.

This paper provides a summary of the ultrasonic pulse technique for pressure sensor validation, and describes the previously applied Prony's method. An introduction to Prism signal processing is then given, followed by a simulation of a Prism-based technique to analyze the ultrasonic signal. A key difference between Prony's method and the Prism technique is that the former provides a single estimate of each of the key parameters - the frequency and exponential decay factor of each frequency component - while the Prism technique provides a sample by sample estimate of each sinusoidal parameter - frequency, amplitude and phase. Finally, initial experimental results applying a Prism-based scheme to ultrasonic data are given, and future work is described.

\section{VIBRATIONAL ANALYSIS OF A PRESSURE TRANSDUCER}

Commonly, pressure transducers intended for industrial applications are constructed as elastic bodies containing a number of mechanical components (Fig. 1), including the sensor diaphragm. Finite element analysis of a transducer design can predict the frequencies of its natural vibration 

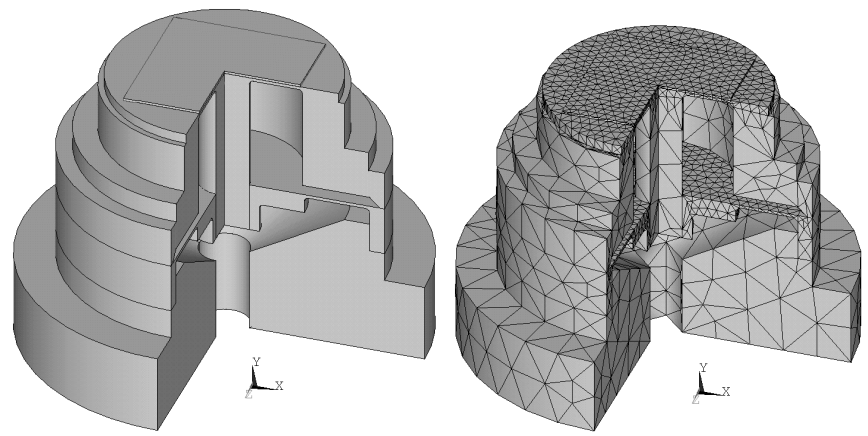

Fig. 1. Pressure transducer structure and finite-element model (from [9])

modes. These frequencies are primarily a function of the mechanical integrity of the transducer, and are generally independent of the industrial operating environment. Thus, sensor defects which may develop over time, such as coating of the diaphragm, may be detected by changes in the resonant frequencies of the transducer.

For example, Fig. 2 shows the results obtained via FE modelling for the frequency response of a particular transducer design under three different scenarios: with a clean diaphragm, and with $0.25 \mathrm{~g}$ and $0.5 \mathrm{~g}$ of added mass (as might be accumulated by fouling of the diaphragm). The figure illustrates several characteristics of the problem. There are several resonant peaks over the range $10-50 \mathrm{kHz}$, and the response of each to the presence of added mass varies significantly. The most obvious change is in the magnitude of response, but in some cases (for example the peak around 45 $\mathrm{kHz}$ ) there are observable frequency shifts too.

The FE modelling results are confirmed by experimental studies, where a prototype transducer is stimulated using ultrasonic pulses to excite its vibratory modes. However, in most practical applications, it would not be possible to provide continuous stimulation of the transducer. Instead, it is envisaged that the device would generate a self-test, consisting of a single ultrasonic burst, followed by a period of signal detection and analysis. These tests would be triggered on an occasional basis, to detect vibrational parameter change over time. However, a single ultrasonic burst will result in an exponentially decaying response at each of the frequencies, which raises the issue of the most appropriate signal processing

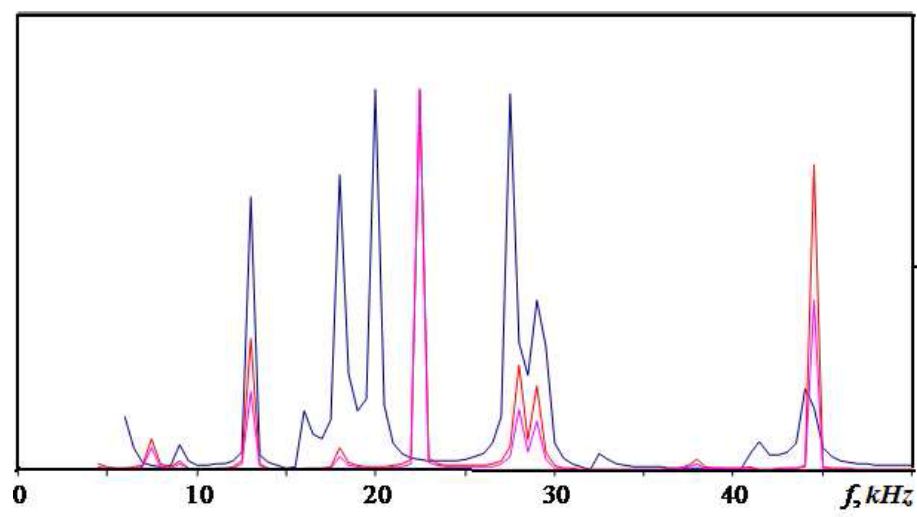

Fig. 2. FE modelled frequency response of pressure transducer: blue - clean diaphragm; red - added 0.25g mass; pink - added 0.5g mass (from [9]) technique to apply in order to detect small changes in modal frequencies and decay rates. Given the limited duration of the transducer response, FFT analysis provides limited frequency resolution, and alternative methods have been sought.

\section{SIGNAL ANALYSIS USING PRONY'S METHOD}

In [10], the SUSU authors present an analysis of Prony's method, adapted for the ultrasonic pressure sensor validation procedure discussed above, in which the optimal sampling rate is selected based on minimizing the condition numbers of the matrices which arise during the calculation.

Prony's method assumes an input signal as the sum of $n$ exponential terms, each with frequency $f_{j}$, initial amplitude $a_{j}$, initial phase $\phi_{j}$, and damping factor $\sigma_{j}$, as follows:

$$
s(t)=\sum_{j=1}^{n} a_{j} e^{-\sigma_{j} t} \sin \left(2 \pi f_{j} t+\phi_{j}\right)
$$

Note that in [10] and elsewhere an equivalent cosine series is used; the sine function is used here for consistency with the Prism notation discussed below.

In its original form, and assuming the absence of noise, Prony's method requires only $4 n$ samples to estimate the four parameter values for each of the $n$ components. The calculation also assumes, therefore, that all parameter values are constant over the data window. However, when noise is present, the basic technique may generate large errors, and additional computational steps are required to reduce noise sensitivity. Improved results may be obtained by optimising one or more of the method's parameters, such as the model order, the number of samples used for the analysis, or the sampling rate.

A three mode $(n=3)$ simulation example is used in [10] to illustrate the benefits of the adaptation to Prony's method. Frequencies $f_{j}$ of $500 \mathrm{~Hz}, 1100 \mathrm{~Hz}$, and $1400 \mathrm{~Hz}$ respectively, are used, with corresponding damping factors $\sigma_{j}$ (and, for convenience, initial amplitudes $a_{j}$ ) of 100, 200 and 250 respectively. The optimal sampling frequency in this case is found to be $3800 \mathrm{~Hz}$, i.e. only about three times the highest frequency component in the signal.

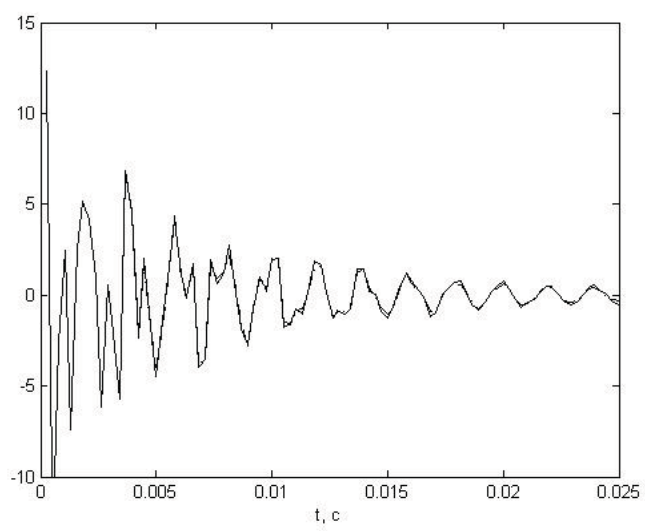

Fig. 3. Noisy signal $(\mathrm{SNR}=30 \mathrm{~dB})$ and its reconstruction using modified Prony method, Fs=3800 Hz (from [10]) 
Fig. 3 shows an example of the original noisy signal and its reconstruction based on the calculated parameter values. Prony's method is an off-line technique, where a least squares fit is sought for the best parameter values for a given data set. The Prism technique, by contrast, is based on a real-time, sample-by-sample approach.

\section{Prism Signal Processing}

The Prism (Fig. 4) is a signal processing object which takes an input time series $s(t)$ and generates up to two output time series $G_{s}(t)$ and $G_{c}(t)$. The Prism can be considered as a pair of tightly coupled FIR filters operating over a window of the input data $s(t)$ of duration $2 / m$, where $m$ is the characteristic frequency of the Prism and one of its design parameters. Unusually, the Prism has a recursive calculation, so that the computation per sample is low, and essentially independent of the Prism sample length. For a pure sinusoidal input

$$
s(t)=A \sin \left(2 \pi f t+\phi_{o}\right)
$$

the Prism outputs are given by the following equations:

$$
G_{s}(t)=A \operatorname{sinc}^{2}(r) \frac{r^{2}}{r^{2}-h^{2}} \sin (\phi(t)-2 \pi r)
$$

and

$$
G_{c}(t)=A \operatorname{sinc}^{2}(r) \frac{h r}{r^{2}-h^{2}} \cos (\phi(t)-2 \pi r)
$$

where the instantaneous phase $\phi(t)=2 \pi f t+\phi_{0} ; r=f / m$, the frequency ratio; $\operatorname{sinc}(\mathrm{x})$ is the normalized sinc function; and $h$ is the harmonic number, a small positive integer and a second design parameter for the Prism. The Prism outputs have linear phase delay, while the gains of $G_{s}(t)$ and $G_{c}(t)$ for $h=1$, labelled $\Gamma_{s}$ and $\Gamma_{c}$ respectively, are shown in Fig. 5; these exhibit a generally low pass characteristic with periodic notches at multiples of $m$, including at DC. Networks of Prisms can be used to perform a range of filtering tasks, including low pass, bandpass and (static) notch filtering.

The utility of the Prism for sinusoidal tracking is apparent from the orthogonal relationship between $G_{s}(t)$ and $G_{c}(t)$ : other than the scaling factor $h / r$, the two outputs form an analytic function from which sample-by-sample estimates of frequency, amplitude and phase may be readily derived. A number of sinusoidal trackers, employing one or more Prisms, apply different mechanisms to solve equations (3) and (4).

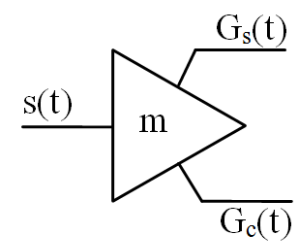

Fig. 4. Prism signal processing block with time series input $s(t)$ as and time series outputs $G_{s}(t)$ and $G_{c}(t)$.
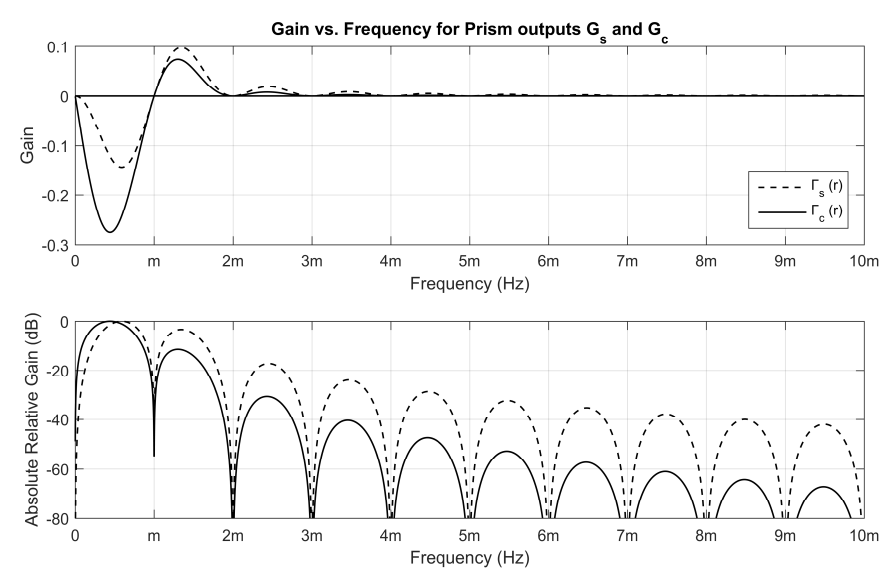

Fig. 5. Gains of Prism outputs $G s(t)$ and $G c(t)$ for $h=1$.

For example, the Recursive Signal Tracker (RST) uses a single Prism and retains recent history of $G_{s}(t)$ and $G_{c}(t)$. From an initial 'guess' (for example the value calculated from the previous sample) of $r$, the signal phase at the current time and at an earlier time are calculated; the phase shift over the known time period gives a revised estimate of $f$ and hence $r, \mathrm{~A}$ and $\phi$. The RST is an efficient sinusoid tracker, operating at signal-tonoise ratios (SNR) of $0 \mathrm{~dB}$ or above without pre-filtering. For example, Fig. 6 shows the ratio of the standard deviation of the frequency, amplitude and phase estimates of the RST outputs, compared to their corresponding Cramér-Rao Lower Bounds (CRLB), where in this case a total of 768 samples are used in the RST data window. For values of $r$ between 0.4 and 0.6 , all parameter estimates fall within a factor of 1.4 of the CRLB. Thus when designing a tracker for an input signal with known frequency range $f$, it is desirable to choose the value of the design parameter $m$ so that $f / m=r \sim 0.5$.

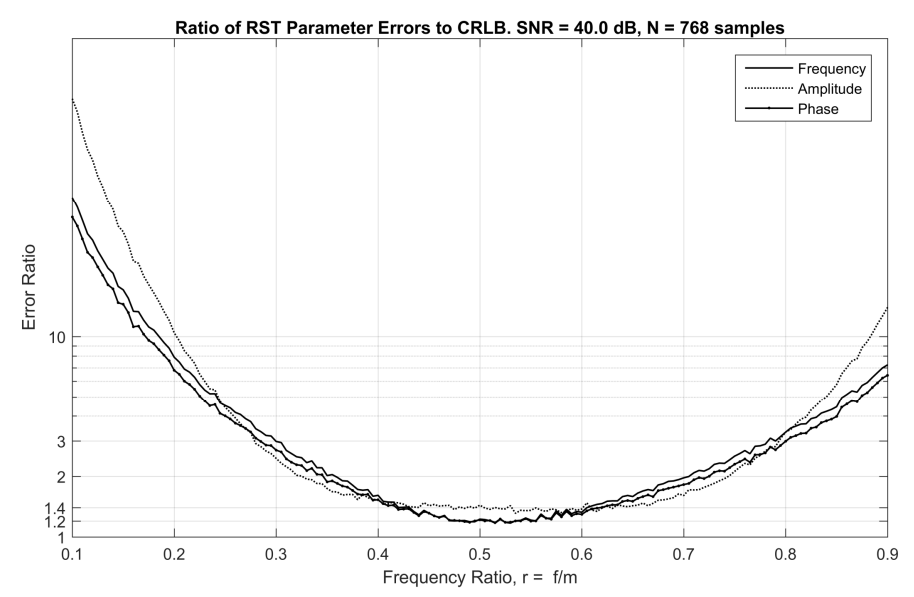

Fig. 6. Recursive Signal Tracker performance v. Cramér-Rao Lower Bound (CRLB), with signal-to-noise ratio of $40 \mathrm{~dB}$ and a 768 sample window. 


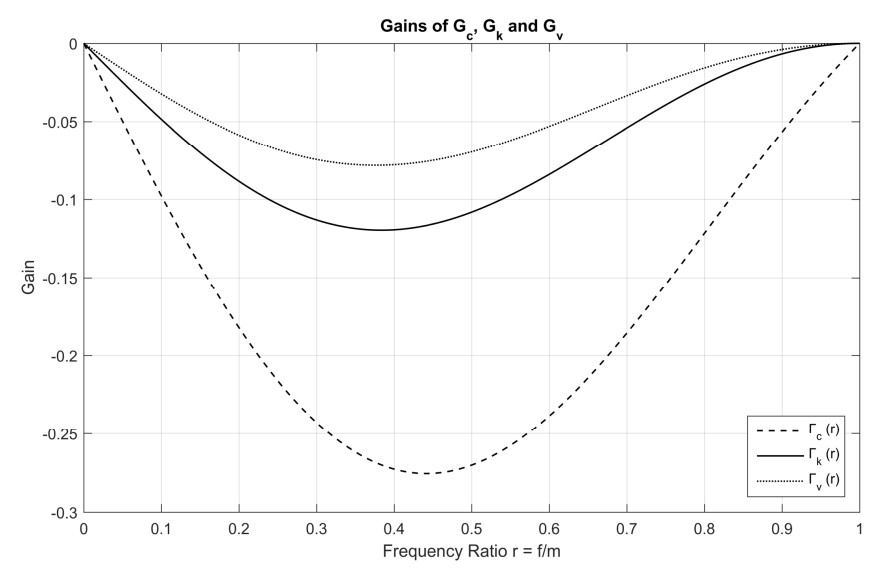

Fig. 7. Gains of Prism outputs $\mathrm{G}_{\mathrm{c}}, \mathrm{G}_{\mathrm{k}}$, and $\mathrm{G}_{\mathrm{v}}$, corresonding to $h=1,2,3$.
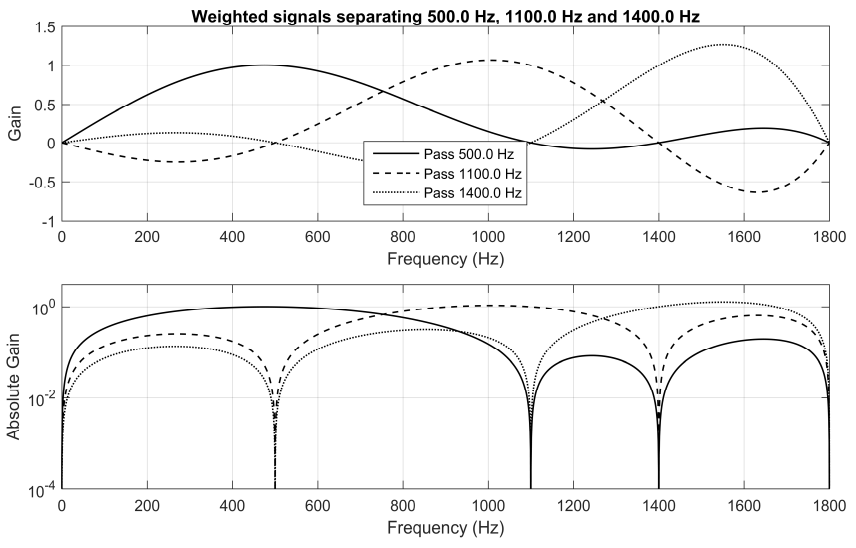

Fig. 9. Weighted combination of Prism outputs: each passes one frequency (from 500,1100 and $1400 \mathrm{~Hz}$ ) with a gain of 1 and notches the other two

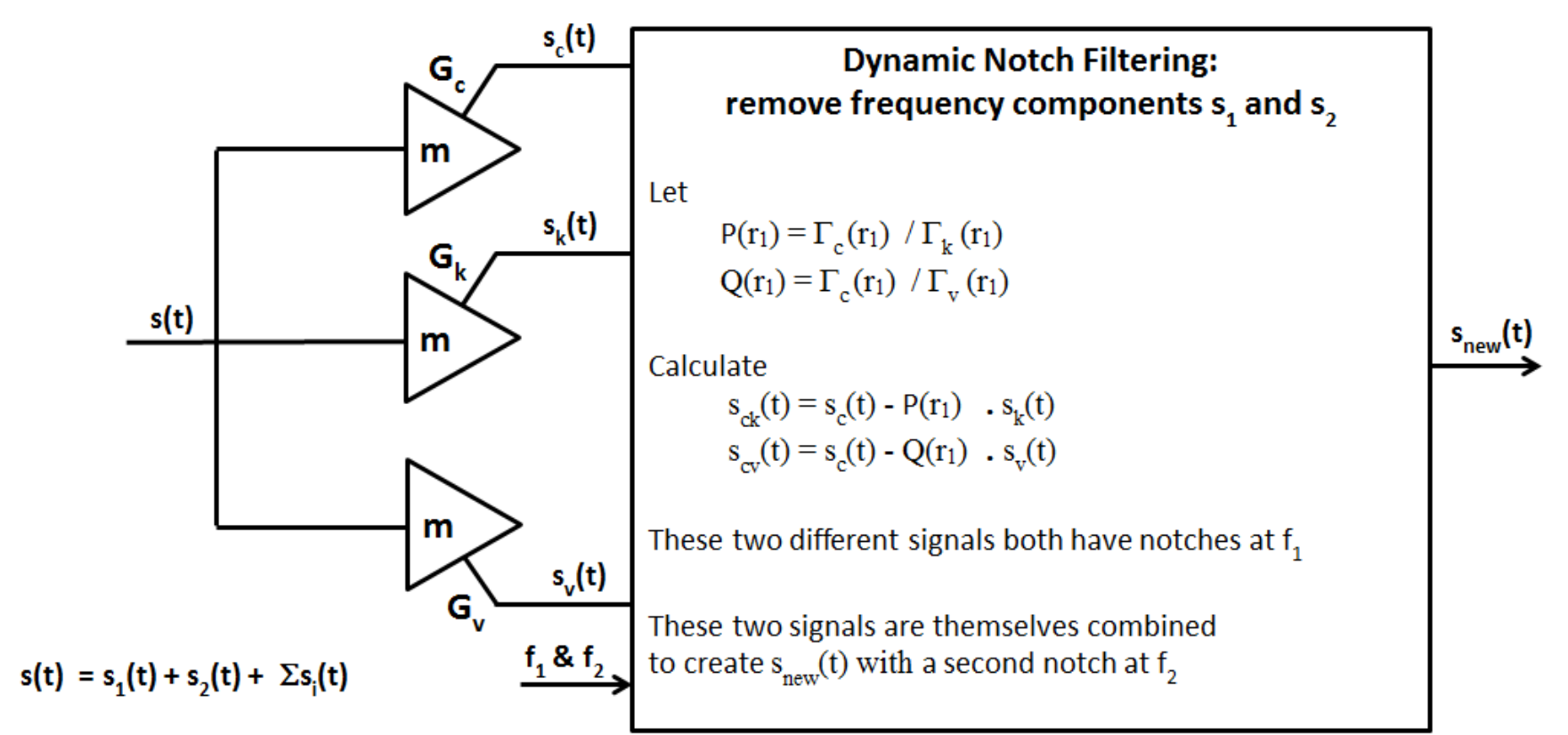

Fig. 8. Dynamic notching of two frequency components

An additional Prism-based technique is Dynamic Notch Filtering (DNF). Given a signal containing several sinusoidal components, DNF facilitiates the removal of one or more such components, where the frequencies to be notched may be selected in real time. This enables the separation and tracking of individual sinusoidal components within the signal. The key is to use two or more Prisms with identical charateristic frequencies $m$ but different harmonic numbers $h$, and where only the cosine outputs $G_{c}$ are generated. From equation (4), for each possible input frequency, the filtered outputs from the Prisms will have the same phase but a different gain, and so a suitably selected weighted sum of the Prism outputs can be used to notch out any desired frequency. As the notch frequency depends only on the weighting of the outputs, not the Prism designs, this can be selected in real time.
Figure 7 shows the gains of Prism cosine outputs for the harmonic numbers $h=1,2$, and 3 respectively, labelled $\Gamma_{c}, \Gamma_{k}$ and $\Gamma_{v}$. These are distinct; there are unique ratios $\Gamma_{c} / \Gamma_{k}$ and $\Gamma_{c} / \Gamma_{v}$ for each frequency ratio $r$. Figure 9 shows a signal processing scheme for notching out any two frequencies $f_{l}$ and $f_{2}$ from the input signal $s(t)$ using three Prisms with common $m$. Suitable weights are calculated based on the gains at the desired notch frequencies (from equation (4)). Although only a single output $s_{\text {new }}(t)$ is shown, the same three Prism outputs can be used with different weightings to generate additional outputs with different notch frequencies. Figure 9 shows how, with a common $m=1800 \mathrm{~Hz}$, the outputs of three Prisms can be combined to notch out any two of the frequencies $500 \mathrm{~Hz}$, $1100 \mathrm{~Hz}$ and $1400 \mathrm{~Hz}$. Thus DNF may be used in the pressure sensor validation as an alternative to Prony's method. 


\section{Prism ANALysis of Pressure TRANSDUCER DATA}

The teams at Oxford and SUSU have begun applying Prism signal processing to the pressure validation problem. A simulation study has been carried out based on equation (1), using the same frequencies $(500,1100,1400 \mathrm{~Hz})$ as in Section III (and Fig. 3), but with exponential decay an order of magnitude slower using $\sigma_{j}=a_{j}=10,20$, and 25. Figure 10 plots the raw input signal and its power spectrum, which indicates the three frequency components present. Figs. $11-$ 13 plot the individual sinusoids separated using Dynamic Notch Filtering along with their power spectra. Figs. 14 and 15 show the values for the frequency and amplitude for the separated $500 \mathrm{~Hz}$ components, generated using an RST, and their errors. Corresponding parameter values (as well as the instantaneous phase) are tracked for the other frequency components but are not shown here for the sake of brevity.

An off-line Prism-based analysis has been applied to experimental data, collected from a pressure transducer subject to ultrasonic probing. A typical time series for the resulting ultrasonic pulse is shown in the upper graph of Fig. 16, while the lower graph shows the corresponding power spectrum. At least nine frequency components are present, ranging in frequency from (approximately) $25 \mathrm{kHz}$ to 145 $\mathrm{kHz}$. Here the data has been sampled at $100 \mathrm{MHz}$ using a 12 bit ADC. Isolation and tracking of the first six components (ordered from the lowest frequency) has been carried out, thus for approximately 25.4, 34.6, 44,5, 58.8, 74.1 and $82.4 \mathrm{kHz}$ respectively.

Given the large number of frequency components in the experiemental data, the technique described above of dynamic notch filtering would be computationally expensive. Instead, given the relatively small variations in frequency expected even when failures occur, static notch filtering can be applied. The insertion in the signal processing path of a Prism with characteristic frequency $m$ creates a notch at every integer multiple of $m \mathrm{~Hz}$. For signals composed of a series of aharmonic components, this presents a straightforward means of isolating each component using a suitably designed Prism pre-filter. This is preferred over, for example, bandpass filtering, which, while perfectly feasible, may restrict the dynamic response and hence the observed decay rates. Static notch filters have been designed for each of the first six frequency components. For example, Fig. 17 shows the filter designed to isolate the second component, at approximately $34.6 \mathrm{kHz}$. The filter envelope has a peak at the desired frequency component while notches are placed at each of the other components to severely attenuate their infleunce in subsequent processing. In the case of the fifth component, at around $74.1 \mathrm{kHz}$, this frequency is close to a multiple of the first component at $25.4 \mathrm{kHz}$. A notch at $25.4 \mathrm{kHz}$ would obscure the $74.1 \mathrm{kHz}$ component, so instead both components are allowed to pass through the static notch filtering and an additional two component dynamic notch filtering scheme is used to isolate the higher frequency component.
Fig. 18 shows the resulting time series for the isolated $34.7 \mathrm{kHz}$ component, along with its power spectrum, both of which suggest the notch filtering design is effective. Figs. 19 and 20 show the RST generated time series for the frequency and amplitude of the same component. While the amplitude time series shows a reasonably smooth decay, the calculated frequency estimate has an oscillatory characteristic which becomes more severe over time as the amplitude of the component (and hence the signal to noise ratio) decreases with time. The extent to which this observed oscillation is a result of the method of calculation, or reflects actual variation of the resonant frequency, is at this stage unresolved.

Four of the six components show broadly similar trends, with oscillating frequency estimates and reasonably smooth decays in the calculated amplitudes. However, the amplitude time series for two of the components, those at 44.5 $\mathrm{kHz}$ and $58.8 \mathrm{kHz}$ respectively, give further evidence of more complex behaviour than the simple exponential decay of fixed frequency sinusoids assumed in Prony's method. The amplitude time series are shown in Figure 21. Both have a distinct change in behaviour around $\mathrm{t}=0.47 \mathrm{~ms}$. Prior to this point in time, the $44.5 \mathrm{kHz}$ component appears to be increasing in value, against basic physical assumptions, while the $58.8 \mathrm{kHz}$ component exhibits a very rapid decline in amplitude. However, after $\mathrm{t}=0.47 \mathrm{~ms}$, the $44.5 \mathrm{kHz}$ component decays in a more conventional manner, while the $58.8 \mathrm{kHz}$ component shows a modest and brief rise, before decaying at a significantly slower rate than in the earlier period of the time series.

Assuming these observed trends (which are visible in all data sets obtained thus far) are at least in part attributable to the actual behaviour of the various vibrational modes of pressure transducer housing (as opposed to an unexpected artifact of the Prism signal processing), one possible explanation is that energy transfer is taking place between the modes i.e. that in the early part of the time sequence the rapid decay in one mode is due to a transfer of energy to the other mode, which as a consequence actually increases its amplitude. An alternative explanation is the influence of reflections and/or secondary ultrasonic pulses. Any of these explnations suggest a more complex physical response to the ultrasonic pulse stimulus, requiring model refinement.

While a more detailed analysis of the physics of the transducer response must be undertaken, alongside extensive further experimentation, we note at this stage in the investigation that the phenomenon has been revealed through the use of Prism signal processing which generates times series of frequency and amplitude values, rather than single point values under the twin assumptions of fixed frequency and explonential decay, as used by Prony's method. Furthermore, these more detailed features (e.g. the time of peak amplitude for the $44.5 \mathrm{kHz}$ component) might provide useful additional diagnostic parameters. 

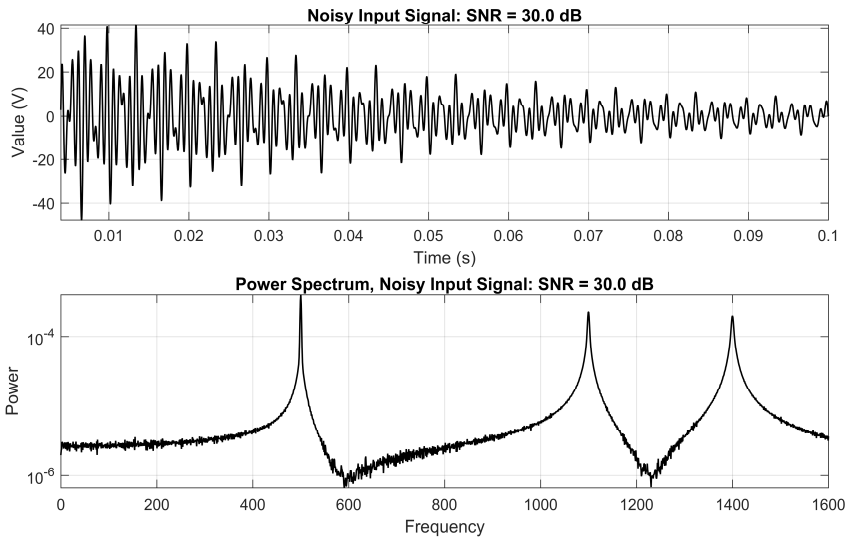

Fig. 10. Simulated input signal with three components
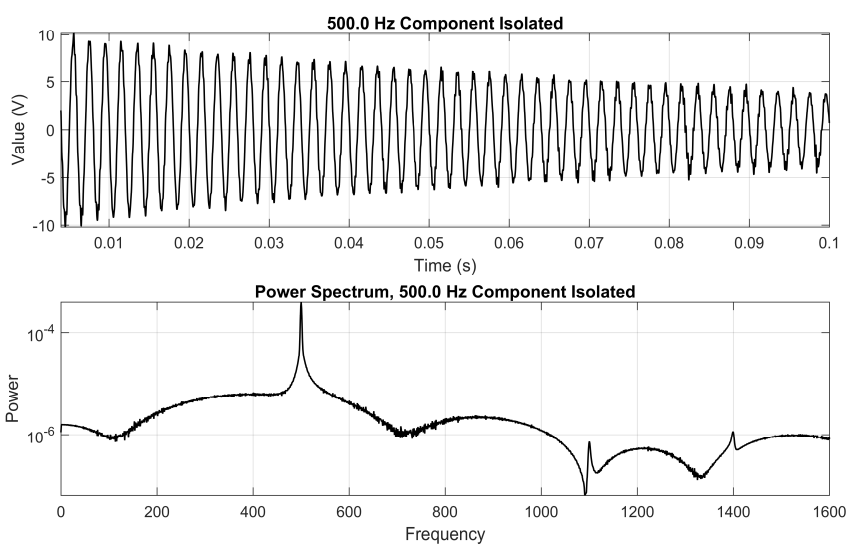

Fig. 11. $500 \mathrm{~Hz}$ component separated using Dynamic Notch Filtering
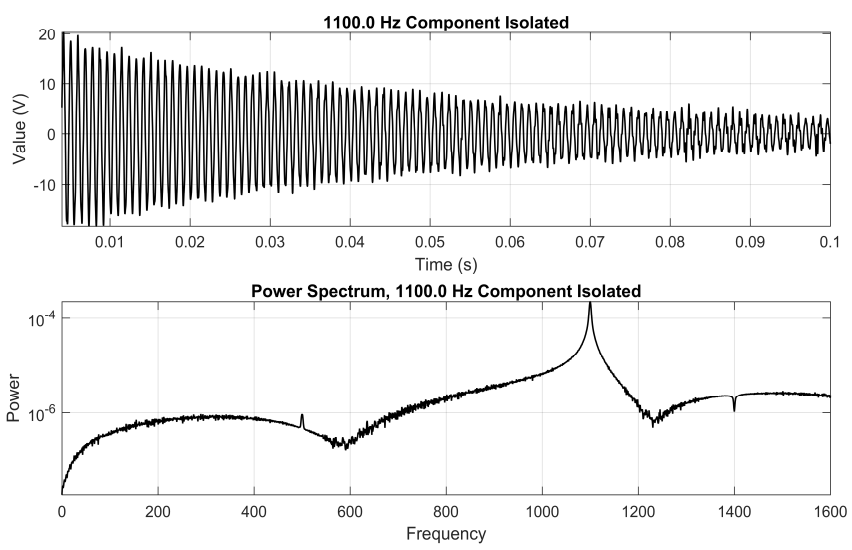

Fig. 12. $1100 \mathrm{~Hz}$ component separated using Dynamic Notch Filtering
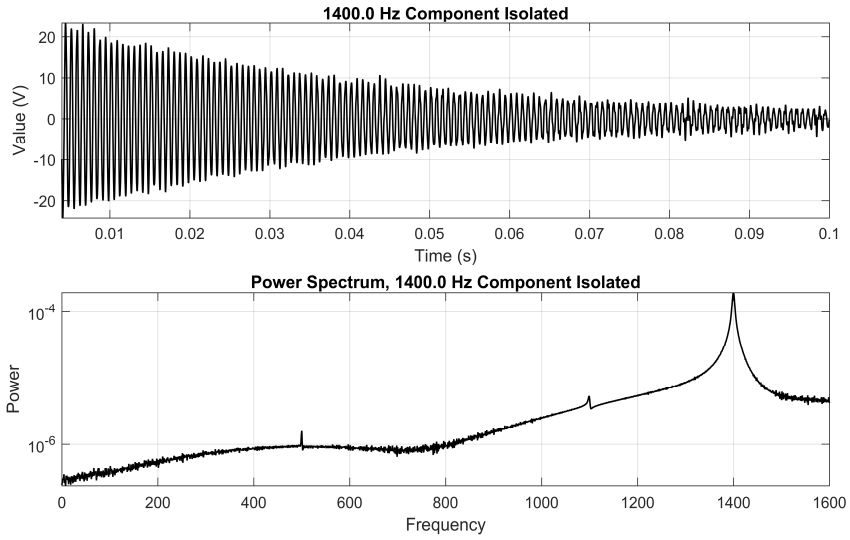

Fig. 13. $1400 \mathrm{~Hz}$ component separated using Dynamic Notch Filtering
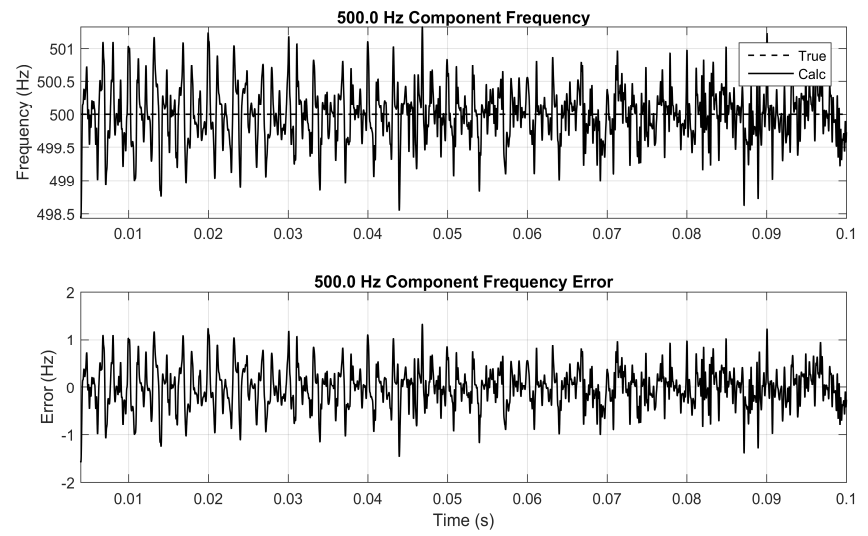

Fig. 14. Frequency tracking on $500 \mathrm{~Hz}$ component
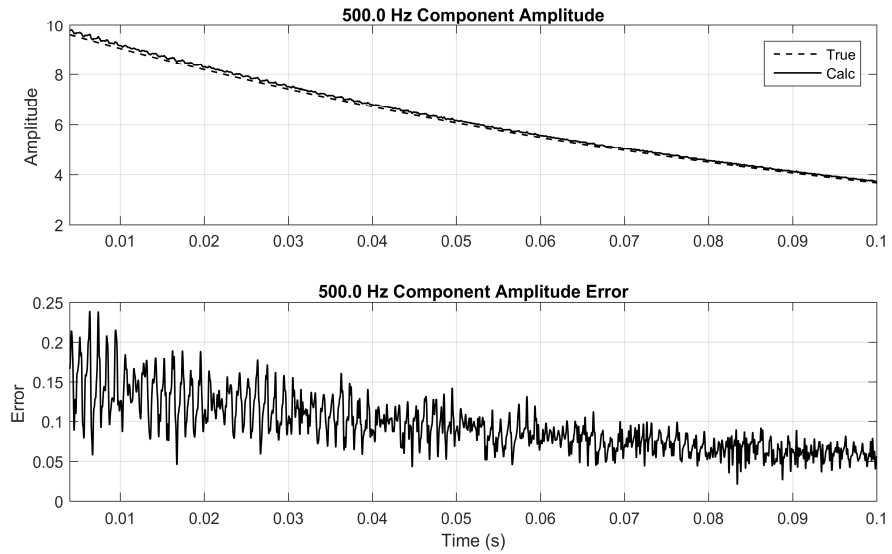

Fig. 15. Amplitude tracking on $500 \mathrm{~Hz}$ component 

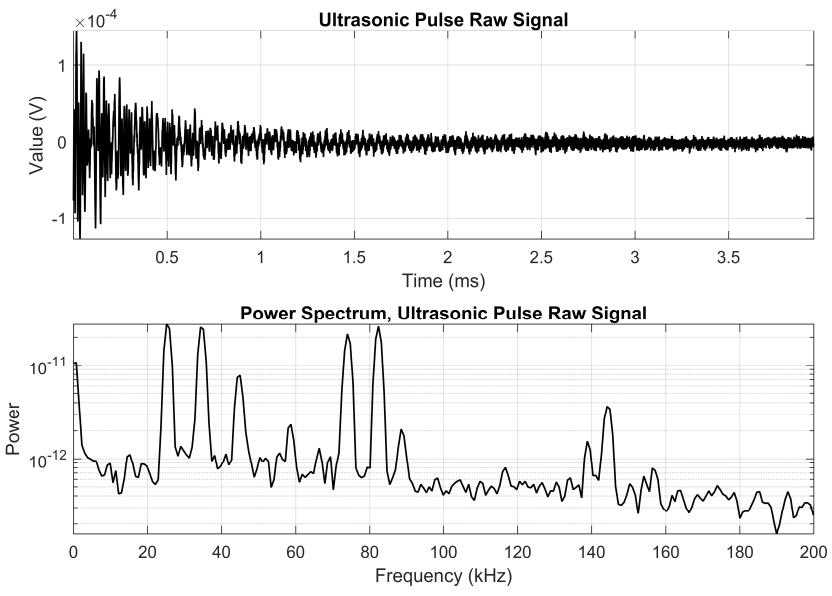

Fig. 16. Time series and spectrum of experimental data

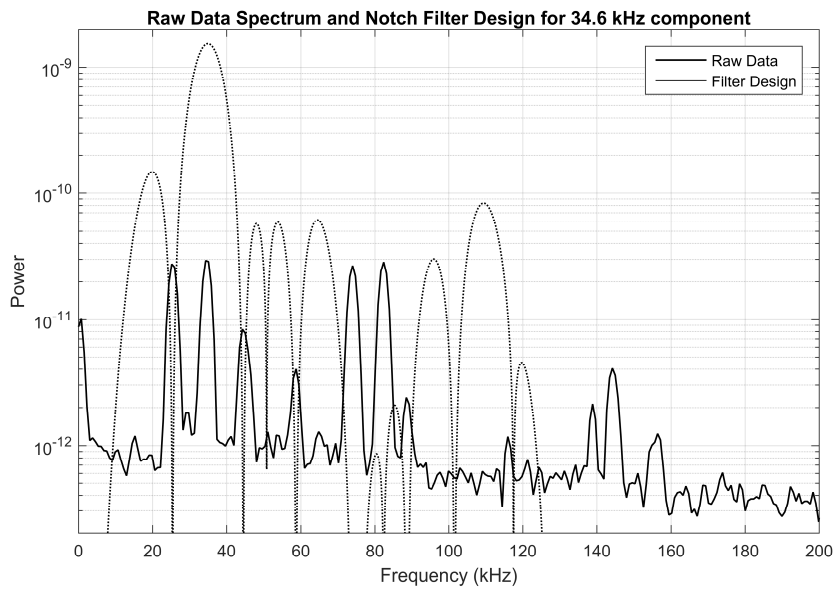

Fig. 17. Prism-based notch filter to isolate $34.6 \mathrm{kHz}$ component
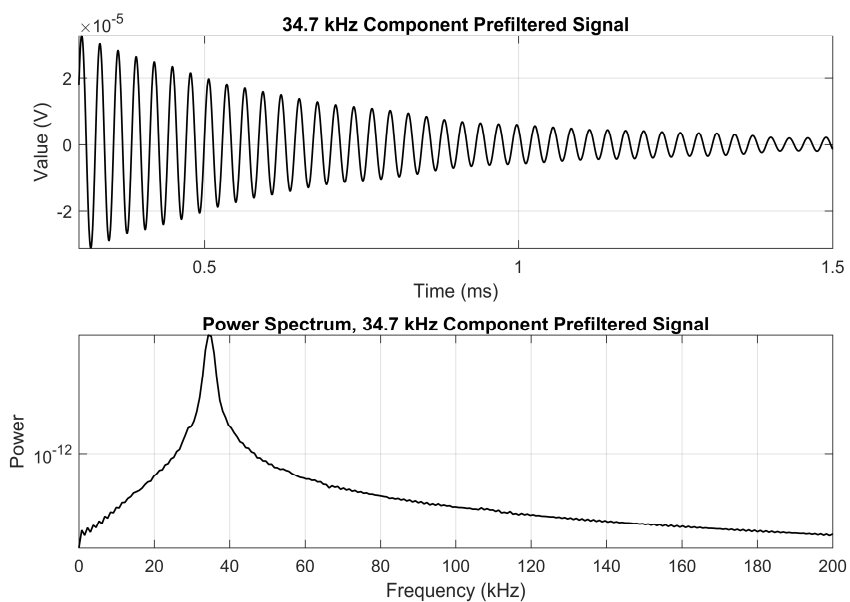

Fig. 18. Time series and power spectrum of isolated $34.6 \mathrm{kHz}$ component

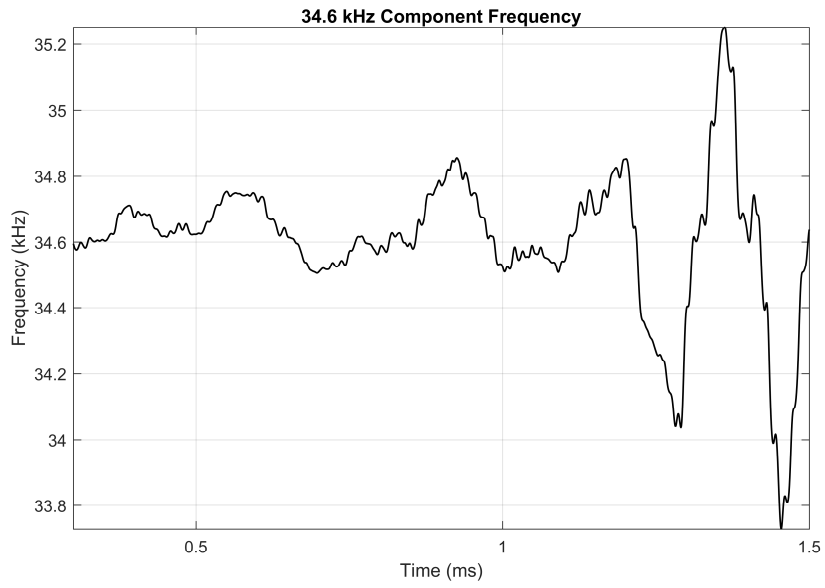

Fig. 19. Time series of frequency estimate for $34.6 \mathrm{kHz}$ component

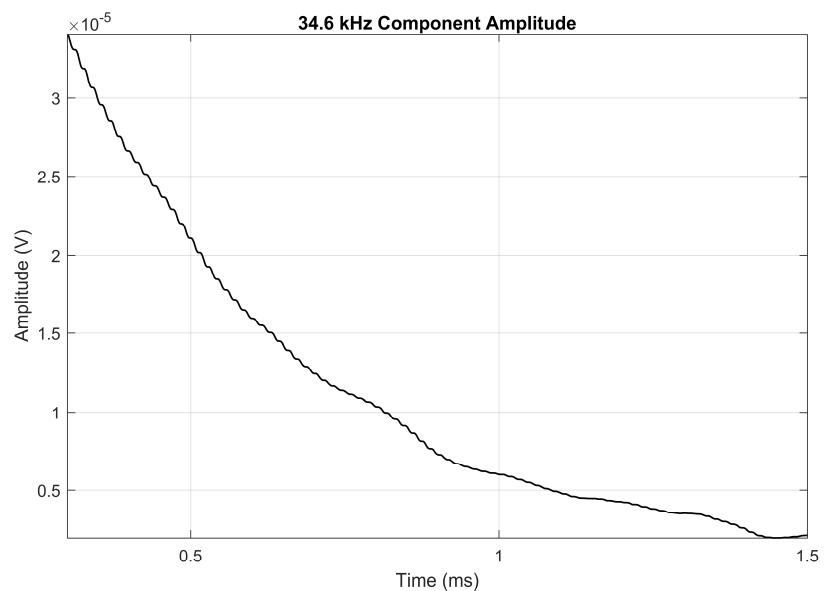

Fig. 20. Time series of amplitude estimate for $34.6 \mathrm{kHz}$ component

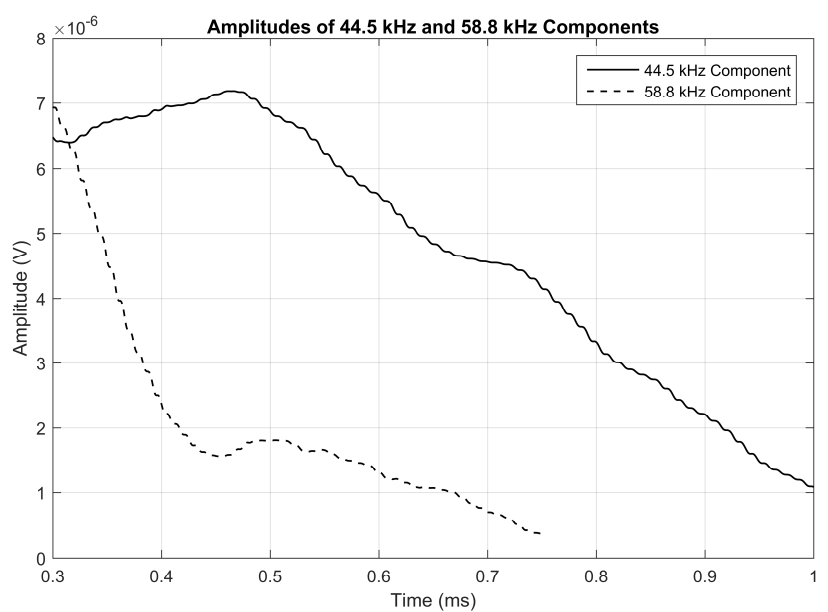

Fig. 21. Time series of amplitude estimates for 44.5 and $58.8 \mathrm{kHz}$ components 


\section{DICUSSION}

The Prism approach contrasts with the Prony technique in several respects. Most obviously, by default it is a (potentially) real-time, single pass method providing sampleby-sample estimates of the sinusoidal component parameter values, whereas Prony's method uses iterative calculations upon a given data set to provide single best estimates of the assumed constant parameter values. Note that only the timevarying amplitude is shown here (Figs 15 and 20) but it is a straightforward matter to further calculate the rate of decay, assuming monotonicity. In providing a times series, the Prism method offers the possibility of more diagnostic detail, as shown in Fig 20 where there are distinct stages in the amplitude behaviour of two of the modes. A more straightforward use of the time series, where an average value of the frequency or decay rate is to be calculated, is to enable an optimal selection of the period over which that calculation is made, trading off a longer data period with increasing relative noise as the signal amplitude drops with time.

The calculation per sample point is low and essentially constant for the Prism technique, but the sampling rate required to achieve the results shown above is relatively high; for accurate results a Prism window typically includes 100 data points or more. In the simulation $230 \mathrm{kHz}$ sampling was used, compared to only $3800 \mathrm{~Hz}$ for the Pronys' method calculation, while in the experimental data a sample rate of $100 \mathrm{MHz}$ was used (lower sampling rates may certainly be feasible). However, arguably a higher data rate is better suited to future technology development. As ADC sampling rates and available computation power continue to improve, signal processing methods that can readily manage higher data bandwidth will be beneficial. In [10], the $3800 \mathrm{~Hz}$ sampling rate was selected for the Prony method over both lower and higher values. Of course, such considerations are also constrained by application requirements - industrial pressure sensors are usually low power, intrinsically safe devices and so a low computational burden is advantageous.

Both the Prism simulation and the experimental analyses require good initial approximate values of the component frequencies. Arguably, this is reasonable for this particular application, as this knowledge should be available: the problem is detecting small changes. However, where a significant change in any frequency is detected, it would be possible to rerun the algorithm using the same data set but with revised best estimates of the component frequencies (or revised static notch filter designs) to improve the quality of the final results.

The most significant limitation of the Prism technique for this application is its ability to manage very rapid amplitude change. The damping factors used in the Prism simulation example were an order of magnitude lower than those for the Prony's method example; using equivalent damping factors resulted in poor parameter tracking. However, the decay rates observed in the experimental data were suited to the Prism technique. Future research will look to develop a new Prism-based tracker capable of following very rapid amplitude change.

The most interesting experimental finding is the suggestion that the amplitude decay is not always monotonic, but rather there appears to be additional physical phenomena causing more complex behaviour. This opens up a whole new vista for more detailed modelling and experimental work, which may in turn suggest improved diagnostic approaches.

\section{REFERENCES}

[1] L.D. Xu, W. He, and S.L. Wang, "Internet of Things in Industries: A Survey", IEEE Transactions on Industrial Informatics, vol. 10, No. 4 pp. 2233-2243, Nov. 2014. DOI :10.1109/TII.2014.2300753.

[2] B. Vogel-Heuser, D. Hess, "Guest Editorial - Industry 4.0-Prerequisites and Visions", IEEE Transactions on Automation Science and Engineering, Vol 14, No. 2, April 2016. DOI: 10.1109/TASE.2016.2523639.

[3] J. O. Kephart and D. M. Chess, "The vision of autonomic computing", IEEE Computer, vol. 36, no. 1, pp. 41-50, Jan. 2003.

[4] M.P. Henry and D.W. Clarke, "The Self-validating Sensor: Rationale, Definitions and Examples", Control Engineering Practice, vol. 1, no. 4, pp. 585-610, 1993.

[5] R.E. Taymanov and K.V. Sapozhnikova, "Metrological selfcheck and evolution of metrology", Measurement, vol.43, no. 7, pp. 869-877, 2010.

[6] G. Monte, V. Huang, P. Liscovsky, D. Marasco, and A. Agnello, "Standard of things, first step: Understanding and normalizing sensor signals," Industrial Electronics Society, IECON 2013 - 39th Annual Conference of the IEEE, Vienna, 2013, pp. 118-123. DOI: 10.1109/IECON.2013.6699121.

[7] Z. Feng, Q. Wang, K. Shida, "A review of self-validating sensor technology", Sensor Review 2007 27:1 , 48-56.

[8] Z. Feng, Q. Wang, and K. Shida, "Design and Implementation of a SelfValidating Pressure Sensor", IEEE Sensors Journal, vol. 9, no. 3, pp. 207-812, March 2009.

[9] O.Yu. Bushuev, A.S. Semenov, A.O. Chernavskiy and A.L. Shestakov, "Detecting changes in the condition of a pressure transucer by analysing its output signal", XX IMEKO World Congress, Busan, 2012.

[10] O. Yu. Bushuev and O. L. Ibryaeva, "Choosing an Optimal Sampling Rate to Improve the Performance of Signal Analysis by Prony's Method", $35^{\text {th }}$ International Conference on Telecommunications and Signal Processing, Prague, 2012. 